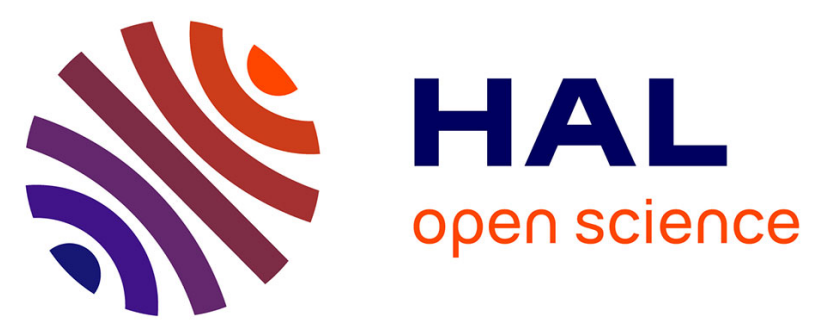

\title{
Multiscale modelling of morphological evolution of rough surface duringsuperficial, volume and evaporation-condensation diffusions
}

\author{
Maxence Bigerelle, J. Favergeon, T. Mathia, A. Iost
}

\section{To cite this version:}

Maxence Bigerelle, J. Favergeon, T. Mathia, A. Iost. Multiscale modelling of morphological evolution of rough surface duringsuperficial, volume and evaporation-condensation diffusions. Defect and Diffusion Forum, 2012, 323-325, pp.101-107. 10.4028/www.scientific.net/DDF.323-325.101 . hal01174144

\author{
HAL Id: hal-01174144 \\ https://hal.science/hal-01174144
}

Submitted on 8 Jul 2015

HAL is a multi-disciplinary open access archive for the deposit and dissemination of scientific research documents, whether they are published or not. The documents may come from teaching and research institutions in France or abroad, or from public or private research centers.
L'archive ouverte pluridisciplinaire HAL, est destinée au dépôt et à la diffusion de documents scientifiques de niveau recherche, publiés ou non, émanant des établissements d'enseignement et de recherche français ou étrangers, des laboratoires publics ou privés. 


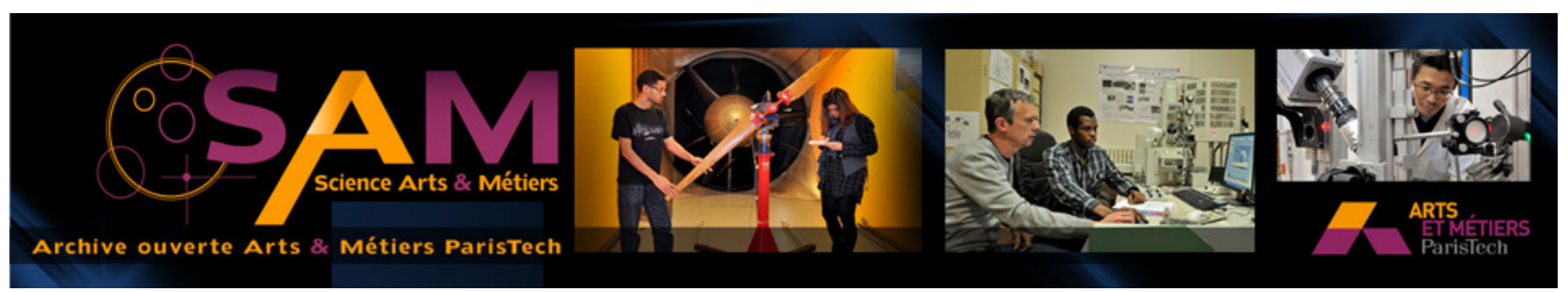

Science Arts \& Métiers (SAM)

is an open access repository that collects the work of Arts et Métiers ParisTech researchers and makes it freely available over the web where possible.

This is an author-deposited version published in: http://sam.ensam.eu

Handle ID: .http://hdl.handle.net/10985/9730

\section{To cite this version :}

M. BIGERELLE, J. FAVERGEON, T. MATHIA, A. IOST - Multiscale modelling of morphological evolution of rough surface duringsuperficial, volume and evaporation-condensation diffusions Defect and Diffusion Forum - Vol. 323-325, p.101-107 - 2012 


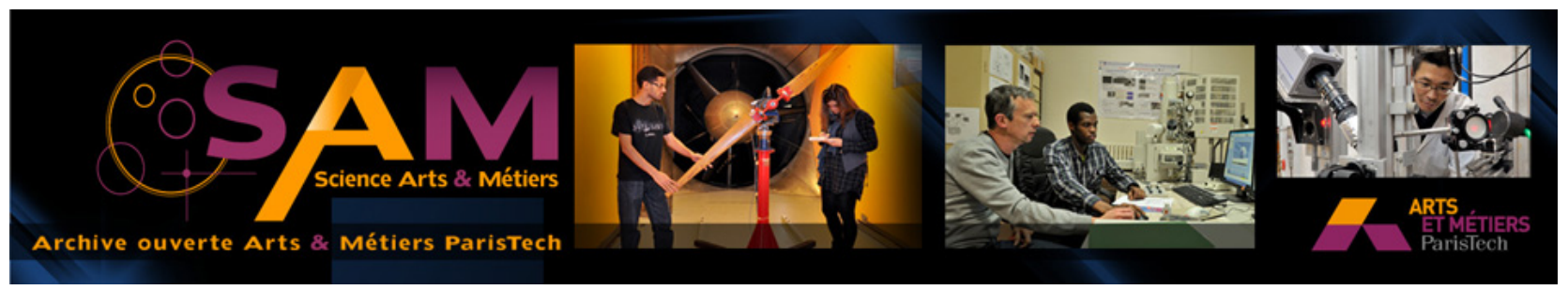

\section{Science Arts \& Métiers (SAM)}

is an open access repository that collects the work of Arts et Métiers ParisTech researchers and makes it freely available over the web where possible.

This is an author-deposited version published in: http://sam.ensam.eu Handle ID: .http://hdl.handle.net/null

\section{To cite this version :}

Maxence M BIGERELLE, J FAVERGEON, Thomas T MATHIA, Alain IOST - Multiscale modelling of morphological evolution of rough surface duringsuperficial, volume and evaporationcondensation diffusions - Defect and Diffusion Forum - Vol. 323-325, p.101-107 - 2012 


\title{
Multiscale modelling of morphological evolution of rough surface during superficial, volume and evaporation-condensation diffusions
}

\author{
M. Bigerelle ${ }^{1,4, a}$, J. Favergeon ${ }^{1, b}$, T. Mathia ${ }^{2, c}$ and A.lost ${ }^{3, d}$ \\ ${ }^{1}$ Laboratoire de Mécanique Roberval, UTC, UMR 6253, 60205 Compiègne, France \\ ${ }^{2}$ Laboratoire de Tribologie et Dynamique des Systèmes, UMR 555, 36, Avenue Guy de Collongue, \\ 69131 Ecully cedex, France \\ ${ }^{3}$ Arts et Metiers ParisTech ; LML CNRS 8107 ; ENSAM, 8 boulevard Louis XIV, 59046 Lille Cedex, \\ France. \\ ${ }^{4}$ UVHC, TEMPO EA 4542, F-59313 Valenciennes, France. \\ amaxence.bigerelle@utc.fr, bjerome.favergeon@utc.fr, 'Thomas.Mathia@ec-lyon.fr, \\ dalain.iost@ensam.eu
}

Keywords: roughness, fractal dimension, superficial diffusion, model.

\begin{abstract}
Fractal functions are used to model a metallic interface. An analytical model described by three partial differential equations is built to model time evolution of the surface during heating including three different mechanisms of diffusion: superficial diffusion (SD), volume diffusion (VD) and diffusion by evaporation-condensation (DEC). Initial topographies are modeled by Stochastic Weierstra $\beta$ functions because of their ability to reproduce experimental roughness profiles. Applied to an aluminum alloy at $550^{\circ} \mathrm{C}$, a high number of roughness parameters and their variance are calculated. A classification method shows that the best geometrical approach that discriminates heat effect is the fractal dimension. The most popular parameter, $\mathrm{R}_{\mathrm{a}}$, badly discriminates processes (classification number $=58$ ). The four order spectral moments of the roughness profile are correlated with the evolution of profile. It is shown theoretically that the superficial diffusion depends directly to the fourth spectral moment of the roughness profile.
\end{abstract}

\section{Introduction}

The fractal concept is of interest for analyzing surface topographies and it is often reported that the fractal parameter describes surface texture in more detail than conventional roughness parameters. Roughness parameters versus the length scale presents some scaling laws and often data yields detailed information on length scale dependent to the surface topography. Fractal functions are used to model a metallic interface and an analytical model is built to model time evolution of the surface during heating including three different mechanisms of diffusion: superficial diffusion (SD), volume diffusion (VD) and diffusion by evaporation-condensation (DEC).

\section{Multi-Physics Models of diffusion on rough surface}

To model the time evolution of surface during annealing, we used the formalism described by Herring [1,2] and Mullins [3,4]. The viscous displacement is neglected because it is very low for metallic surfaces if they are not constrained. We supposed that there are no foreign atoms at the interface (vacuum heating). The surface diffusion is driven by surface curvature gradients. The surface self-diffusion flux of atoms $J_{\mathrm{s}}$, due to capillarity forces is everywhere proportional to the gradient of the chemical potential. The diffusion fluxes induce a variation of the surface profile and the kinetics of a surface element along the outward normal to the surface. By taking the surface divergence of $\mathrm{J}_{\mathrm{s}}$, multiplied by the atomic volume, a basic differential equation describing the profile variation by surface diffusion is obtained ( $\mathrm{SD}$, Table 1). The evaporation rate over a clean surface is given by the Langmuir equation, derived from the kinetic gas theory. In the particular case of evaporation in vacuum, the incident flux of surrounding atoms toward the surface is negligible compared to the outward flux of the atoms from the surface to the vacuum. The net evaporization flux 
$\mathrm{J}_{\mathrm{e}}$ is then obtained. The vapor pressure over a curved surface is related to the vapor pressure over a flat surface given by the Gibbs-Thompson equation which means that the evaporation flux increases as the local curvature increases. This evaporation flux causes a depletion of the surface profile and the related kinetics of a surface element along outward normal to surface is obtained by multiplying the flux $\mathrm{J}_{\mathrm{e}}$ by the atomic volume and the differential equations is then obtained (EVD, Table 1). Concerning the last mechanism, Furthermore, the concentration of vacancies at the surface of a crystalline particle strongly depends on surface stresses that can be quantified with curvature. This gives rise to a distribution in the concentration of vacancies around any shape withnon-uniform curvature. The concentration of vacancies in the neck region is much higher than those vacancy concentrations on the particle surface faraway from the neck, which provides a driving force for diffusion.Vacancies move out of the neck region, either by diffusing through the interior lattice (termed bulk or volume diffusion). The chemical potential of a crystalline material can be well approximated by the concentration of vacancies, and the driving force for the volume diffusion of vacancies can then be formulated according to Fick's law. A balance equation is then written in quasi-steady-state dimensionless. Differences in curvature along the particle surface produce stresses that subsequently produce differences in chemical potential. These chemical potential differences are translated to vacancy concentration differences. The resulting relation between mean curvature and vacancy concentration along the surface yields the celebrated Gibbs-Thomson equation. Then the final differential equation is obtained (VD, Table 3). Finally, Under local equilibrium hypothesis between surface and volume and supposing that surface energy $\gamma$ is space independent, diffusion could be modeled by three partial differential equations (Table 1) where $\Omega$ is atomic volume, $\gamma_{\mathrm{s}}$ the surface energy, $R$ the radius of curvature, $n_{s}$ the density of surface sites, $D_{s}$ the surface self-diffusion coefficient, $\mathrm{k}$ the Boltzmann's constant, $\mathrm{T}$ the absolute temperature, $\mathrm{z}$ the height of profiles, $\mathrm{D}_{\mathrm{V}}$ the volume diffusion coefficient, $\mathrm{P}_{0}$ the vapor pressure and $\mathrm{M}$ is the atomic mass. Traditionally, for this approach CGS system is used.

\begin{tabular}{|l|l|l|l|}
\hline \multicolumn{4}{|c|}{ Table 1. Partial derivative equations used to model interfacial diffusion. } \\
\hline Diffusion & $\mathrm{SD}$ & $\mathrm{VD}$ & $\mathrm{EVD}$ \\
\hline $\begin{array}{l}\text { Partial } \\
\text { Differential } \\
\text { Equations }\end{array}$ & $\frac{\partial \mathrm{z}}{\partial \mathrm{t}}=-\mathrm{B} \frac{\partial^{4} \mathrm{z}}{\partial^{4} \mathrm{x}}$ & $\frac{\partial 1 / \mathrm{R}}{\partial \mathrm{t}}=-\mathrm{C} \frac{2}{\pi} \int_{0}^{\infty} \mathrm{w}^{3} \cos (\mathrm{wx}) \mathrm{dw} \int_{0}^{\infty} \frac{1}{\mathrm{R}(\xi, \mathrm{t})}$ & $\frac{\partial \mathrm{z}}{\partial \mathrm{t}}=-\mathrm{A} \frac{\partial^{2} \mathrm{z}}{\partial^{2} \mathrm{x}}$ \\
& $\mathrm{B}=\frac{\Omega^{2} \mathrm{n}_{\mathrm{s}}}{\mathrm{kT}} \mathrm{D}_{\mathrm{s}} \gamma_{\mathrm{s}}$ & with $\frac{1}{\mathrm{R}}=-\frac{\partial^{2} \mathrm{z}}{\partial^{2} \mathrm{x}} \mathrm{C}=\frac{\Omega \gamma \mathrm{D}_{\mathrm{v}}}{\mathrm{kT}}$ & $\mathrm{A}=\frac{\Omega^{2} \gamma_{\mathrm{s}} \mathrm{P}_{0}}{(\mathrm{kT})^{\frac{3}{2}} \sqrt{2 \pi \mathrm{M}}}$ \\
\hline
\end{tabular}

To solve these equations, it is necessary to introduce an initial profile at $t=0$. Stochastic Weierstrass function [5,6] are used because their good agreement with experimental roughness profiles. Although Weierstrass function is nowhere derivative, it possesses all derivative components. It is then possible to apply partial differential equations on each component and then obtain a new fractal function. Each component $\mathrm{z}_{\mathrm{n}}(\mathrm{x}, 0)$ of Table 1 is modified during heating process and gives a new component $z_{n}(x, t)$. The values of $z_{n}(x, t)$ is calculated by finding solution with separate variables $\mathrm{z}_{\mathrm{n}}(\mathrm{x}, \mathrm{t})=\Phi(\mathrm{x}) \Psi(\mathrm{t})$ and supposing that profiles modification is due to the independent and simultaneous action of the three diffusion processes (SD, VD, EVD). After applying the calculations (see appendix 1), by summing all components of the Weierstrass function, one obtains the following equations that characterizes the time evolution of profiles:

$$
z(x, t)=\sum_{n=0}^{\infty} a_{n} \omega^{-n H} \cos \left(\omega^{n} x+\phi_{n}\right) \exp \left(-\left(B \omega^{4 n}+C \omega^{3 n}+A \omega^{2 n}\right) t\right)
$$


Where $\mathrm{z}(\mathrm{x}, 0)$ represents the initial profile $\mathrm{z}(\mathrm{x}, \mathrm{t})$ at diffusion time $\mathrm{t}=0, \mathrm{H}$ the Hölder exponent $(H \in[0,2 \pi]), \omega$ a pulse with $\omega>1, a_{n}$ normalized Gaussian random numbers and $\phi_{n}$ uniform random numbers bounded in $[0,2 \pi]$.

This equation shows that amplitude decays exponentially. For each component, the decay is governed by three time constants characteristic of each diffusion mechanism (figure 1).

$$
\tau_{\mathrm{A}, \mathrm{n}}=\left(\frac{\lambda_{\mathrm{n}}}{2 \pi}\right)^{2} \frac{1}{\mathrm{~A}}, \quad \tau_{\mathrm{B}, \mathrm{n}}=\left(\frac{\lambda_{\mathrm{n}}}{2 \pi}\right)^{4} \frac{1}{\mathrm{~B}} \quad \tau_{\mathrm{C}, \mathrm{n}}=\left(\frac{\lambda_{\mathrm{n}}}{2 \pi}\right)^{3} \frac{1}{\mathrm{C}}
$$

Where $\lambda$ is the wavelength $\left(\omega^{\mathrm{n}}=2 \pi / \lambda_{\mathrm{n}}\right)$. Threshold critical wavelength are given by $\lambda_{\mathrm{B} \Leftrightarrow \mathrm{C}}$, (threshold volumic diffusion and superficial diffusion) and $\lambda_{\mathrm{C} \Leftrightarrow \mathrm{A}}$ (threshold volumic diffusion and evaporation-condensation).

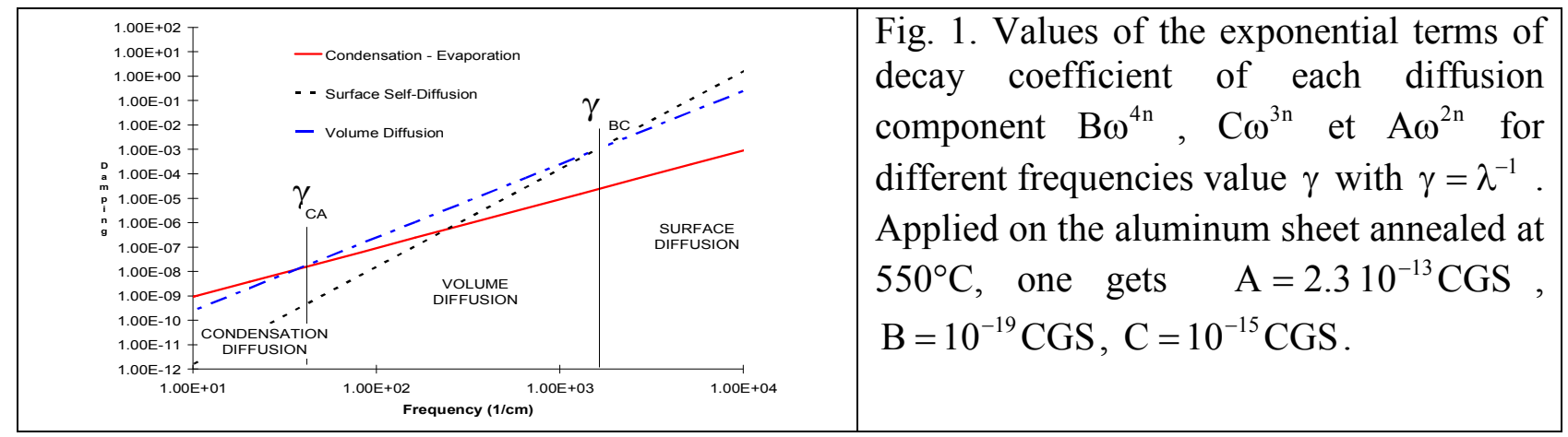

A low time constant implies a high exponential decay and more important is the diffusion mechanism. When profile wavelength increases (lower harmonics in Eq. (1)), then time constant increases and high frequency component decreases in amplitude. However, fractal aspect of profile is given by these frequencies and then the superficial diffusion (adatoms and advacancies motions) will be responsible of the "loss of fractality" of profile. Equation (1) contains, for lower n, a preponderant decay term given by $\mathrm{A}$, for middle $\mathrm{n}$ the decay is given by $\mathrm{C}$ and by $\mathrm{B}$ for greater $\mathrm{n}$.

\section{Theoretical fractal dimension of diffusion processes}

In this part, it will be shown that the fractal dimension will be equal to unity when the diffusion processes are active $(t>0)$. In fact fractal dimension becomes unchanged if the $p$ first terms (lower frequency components) are suppressed ( $\mathrm{p}$ is chosen to respect the following inequality: $\left.\mathrm{B} \omega^{4 \mathrm{p}}>>\mathrm{C} \omega^{3 \mathrm{p}}\right)$. Missing out Gaussian amplitudes that leave fractal dimension of profile unchanged $[7,8]$, equation (2) becomes:

$$
z_{n}^{\prime}(t, x)=\sum_{n=p}^{\infty} \omega^{-n H} \cos \left(\omega^{n} x\right) \exp \left(-B \omega^{4 n} t\right)
$$

Naively it might be expected that a continuous function must have a derivative, or that the set of points where it is not differentiable should be "small" in some sense. This might be because it is difficult to draw or visualize a continuous function whose set of non differentiable points is something other than a finite set of points. The Weierstrass function could perhaps be described as one of the very first fractal studied, although this term was not used until much later. The function has detail at every level, so zooming in on a piece of the curve does not show it getting progressively closer and closer to a straight line. Rather between any two points no matter how close, the function will not be monotone. To obtain this fractal aspect, signal frequencies $\omega^{\mathrm{n}}$ must not decrease quicker than amplitude because final component will vanish and leads to a non fractal function (i.e. classical Fourier series).

let:

$$
\begin{array}{cccc}
\mathrm{G}= & \omega^{-\mathrm{nH}} \exp \left(-\mathrm{B} \omega^{4 \mathrm{n}} \mathrm{t}\right) & \times & \omega^{\mathrm{n}} \\
\mathrm{G}= & \text { amplitude } & \times & \text { frequency }
\end{array}
$$


Following condition must be respected $\lim _{\mathrm{n} \rightarrow \infty} \mathrm{G}=\infty$ otherwise fractal dimension of $\mathrm{z}_{\mathrm{n}}^{\prime}(\mathrm{t}, \mathrm{x})$ will be equal to unity. Then

$$
\mathrm{G}=\exp \left(\mathrm{n} \log \omega(1-\mathrm{h})-\mathrm{B} \omega^{4 \mathrm{n}} \mathrm{t}\right)
$$

As $\omega>1$, then

$$
\left\{\begin{array}{l}
\lim _{n \rightarrow \infty} G=\infty \Leftrightarrow t=0 \\
\lim _{n \rightarrow \infty} G=0 \Leftrightarrow t>0
\end{array}\right.
$$

For $\mathrm{t}=0$, fractal dimension is given by $\Delta=2-\mathrm{H}$. As far back as superficial diffusion is active $(t>0)$, profile loses its fractality (any diffusion process or coupled diffusion processes will lead to same conclusion).

Some defects could be formulated to our approach:

- Herring's equations model macroscopic phenomena meaning that the time must be great with regards to the frequencies jump $\Gamma_{\mathrm{s}}$ of $(\mathrm{ad})$ atoms and $(\mathrm{ad})$ vacancies: $t>>1 / \Gamma_{\mathrm{s}}$.

- Herring's equations suppose that profiles are derivative down to four orders. However, Weierstrass function gets no derivative.

These remarks show that profile can be considered as fractal on a measure range $\left[\mathrm{d}_{\min }, \mathrm{d}_{\max }\right]$ where Weierstrass function models well experimental profiles and where Herring's equations well describe phenomenological diffusion processes. Under these hypotheses, Weierstrass' function can be seen mathematical derivative because of the finite number of components. Fractal dimension is then being considered as a local fractal dimension.

\section{Diffusion modeling on aluminum}

Applied on the aluminum sheet annealed at $550^{\circ} \mathrm{C}$, one obtains the numerical data: $\mathrm{D}_{\mathrm{s}}=10^{-4} \mathrm{~cm}^{2} / \mathrm{s}$, $\mathrm{D}=10^{-8} \mathrm{~cm}^{2} / \mathrm{s}, \mathrm{B}=10^{-19} \mathrm{CGS}, \mathrm{C}=10^{-15} \mathrm{CGS}, \mathrm{M}=23, \Omega=1.1810^{-23}, \mathrm{~A}=2.310^{-13} \mathrm{CGS}$. thanks to the Shannon's theorem, the highest frequency is the half of the critical minimal length that is given by the lattice parameter $\mathrm{a}=510^{-8} \mathrm{~cm}$ and leads to $\mathrm{n}_{\mathrm{c}}=44$. One retains the following Weierstrass function parameters: $\omega=2, \mathrm{t} \in\left[1 . .2^{30}\right\rfloor \mathrm{s}, \mathrm{x} \in[0 . .0 .1] \mathrm{cm}$ et $\mathrm{H}=0 \quad(\Delta=2)$; we have first calculated the values of the exponential term for each component of diffusion $\left(B \omega^{4 n}, C \omega^{3 n}\right.$ et $\left.A \omega^{2 n}\right)$ at different frequencies (Fig. 1). Threshold critical wavelength are given by $\lambda_{\mathrm{B} \Leftrightarrow \mathrm{C}}=8 \mu \mathrm{m}$, $\left(\gamma_{\mathrm{B} \Leftrightarrow \mathrm{C}}=1300 \mathrm{~cm}^{-1}\right)$ (threshold volumic diffusion and superficial diffusion) and $\lambda_{\mathrm{C} \Leftrightarrow \mathrm{A}}=400 \mu \mathrm{m}$ $\left(\gamma_{C \Leftrightarrow A}=24 \mathrm{~cm}^{-1}\right)$ (threshold volumic diffusion and evaporation-condensation). During heating, diffusion will be active by evaporation-condensation for wavelength of profiles greater than $400 \mu \mathrm{m}$, by volumic diffusion for wavelength lying between 8 and $400 \mu \mathrm{m}$ and by superficial diffusion for wavelength lower than $8 \mu \mathrm{m}$. The exponential terms shows decay from the higher frequencies to the lower frequencies. Evolution during heating of a profile of $0.1 \mathrm{~cm}$ length and $\Delta=2$ fractal dimension is shown on Fig. 2 and it can be seen that the profile becomes smoother when the annealing time increases.

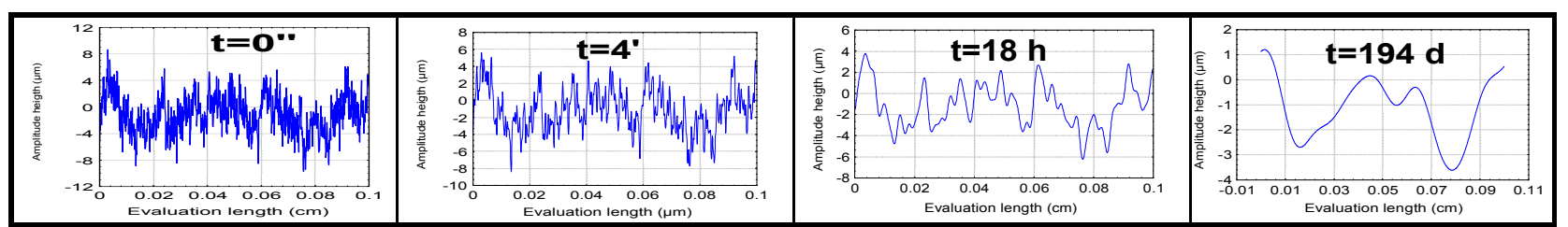

Fig. 2. Evolution of the surface topographies of aluminum interface simulated with a Weierstrass function with fractal dimension of $\Delta=2$. This interface is submitted to a superficial diffusion processes, a volumic one and an evaporation-condensation. Heating temperature is $550^{\circ} \mathrm{C}$, profile height is measured in $\mu \mathrm{m}$ and scanning length in $\mathrm{cm}$. Heating time increases according to the power $2^{p}$ seconds, $(p \leq 30)$. Curves are discretized in 1000 points. (Notations: " : second, ' : minute, h : hour, d : day). 


\section{Roughness parameter calculation}

It was shown theoretically that fractal dimension is equal to 1 as soon as diffusion processes are active. However, what about the numerical estimation of the fractal dimension? What are the roughness parameters that best discriminate the effect of heating on the substrate? To answer these questions, a high number of roughness parameters used in the characterization of the surface topography are calculated. For each roughness parameter, a variance analysis is performed. The Fisher variable $\mathrm{F}$ is then considered as to be the variance of the roughness parameter estimation for all heating time divided by the variance of the same roughness parameter for each heating time. The greater $F$ is, the more discriminate is the roughness parameters with regards to the annealing time. It could then be concluded that the roughness parameter very well characterizes the diffusion processes. By descending ordering $\mathrm{F}$ values, it could then be possible to classify roughness parameters by the power of discrimination. An original bootstrap technique allows us to give a $95 \%$ confidence interval for each $\mathrm{F}$ value $[9,10]$. This classification shows (Fig. 3) that the best parameter is the fractal dimension (classification number $1, \mathrm{~F}=95356$ ). The most popular and used parameter $R_{a}$ badly discriminates processes (classification number $=58, \mathrm{~F}=24$, see fig 3.). The four order spectral moment $\mathrm{m}_{4}=\left\langle\frac{\partial^{4} \mathrm{z}}{\partial^{4} \mathrm{x}}\right\rangle=\int_{0}^{\infty} \gamma^{4} \mathrm{P}\left[\hat{\mathrm{z}}_{\gamma, \mathrm{t}}\right] \mathrm{d} \gamma$ where $\mathrm{P}\left[\hat{\mathrm{z}}_{\gamma, \mathrm{t}}\right\rfloor$ is the spectra, is correlated with the evolution of profile with $\partial z / \partial t=-\mathrm{Bm}_{4}$. To compute $\mathrm{m}_{4}$, the Power Spectral Density $\mathrm{P}\left[\hat{\mathrm{z}}_{\gamma, \mathrm{t}}\right\rfloor$ is firstly determined using a classical Fast Fourier Transform and then a numerical summation is applied to compute this spectral moment. Then, effect of the superficial diffusion depends directly to the four moments and after computations, one obtains the following theoretical values:

$$
\left\langle\frac{\partial^{4} \mathrm{Z}}{\partial^{4} \mathrm{x}}\right\rangle=\frac{\mu_{\mathrm{Z}}^{2}}{4 \log \omega}\left[\frac{\left(8 \mathrm{tA} \pi^{2}\right)^{\mathrm{H}-2} \Gamma(2-\mathrm{H})}{2}+\frac{\left(16 \mathrm{Ct} \pi^{3}\right)^{\frac{2}{3}(\mathrm{H}-2)} \Gamma\left(\frac{2}{3}(2-\mathrm{H})\right)}{3}+\frac{\left(32 \mathrm{tB} \pi^{4}\right)^{\frac{1}{2}(\mathrm{H}-2)} \Gamma\left(\frac{1}{2}(2-\mathrm{H})\right)}{4}\right]
$$

This equation shows that the evolution of $\mathrm{m}_{4}$ depends on time and the fractal dimension of profiles. This parameter calculated by Fast Fourier Transform is classified on position 15 with a F value of 760 . From these results, any remarks can be drawn:

- Calculated fractal dimensions tend to 1 when the time increases: why numerical fractal dimension is not equal to unity for $t>0$ as it was theoretically shown? The demonstration we made supposes that profile frequencies are infinite in the beginning of the diffusion processes, meaning that the amplitude of higher harmonics decays. Then we have calculated the value of the theoretical spectra (see appendix 2):

$$
\mathrm{P}[\hat{\mathrm{z}}(\gamma, \mathrm{t})]=\frac{\mu_{\mathrm{Z}}^{2}}{4 \gamma^{2 \mathrm{H}+1} \log \omega} \exp \left(-2 \mathrm{t}\left(\mathrm{A}(2 \pi \gamma)^{2}+\mathrm{C}(2 \pi \gamma)^{3}+\mathrm{B}(2 \pi \gamma)^{4}\right)\right)
$$

This expression shows that a cut off appears for high frequencies and this frequency threshold increases exponentially with time. For $\mathrm{t}=0$ spectrum follows the power law $\mathrm{P}[\hat{\mathrm{z}}(\gamma, \mathrm{t})] \propto 1 / \gamma^{2 \mathrm{H}+1}$ a well-known relation used to calculate the fractal dimension of profiles [7,11]. However, when diffusion is processed, this relation could not be used to calculate the fractal dimension. According to the Shannon's theorem, the component frequencies greater than twice the sampling rate do not affect the calculation of fractal dimension. When frequencies are lower than twice the sampling rate decays, then calculated fractal dimension are affected and decreases monotonically with time. This remark shows that the calculated fractal dimension is always a local fractal one.

Bootstrap analyses of variance shows that the fractal dimension computed by the ANAM method $[12]$ is the best parameter to characterize the diffusion process. The first reason is that ANAM method is statistically a robustness parameter because its three integrations that accordingly to the central limit theorem decreases its variance estimation. The second reason is that $\left\langle\frac{\partial^{4} z}{\partial^{4} x}\right\rangle=f\left(t^{\lambda(H-2)}\right)$ means that the morphology depends both on time and fractal dimension of the initial profile (except for $\Delta=1$ ). 

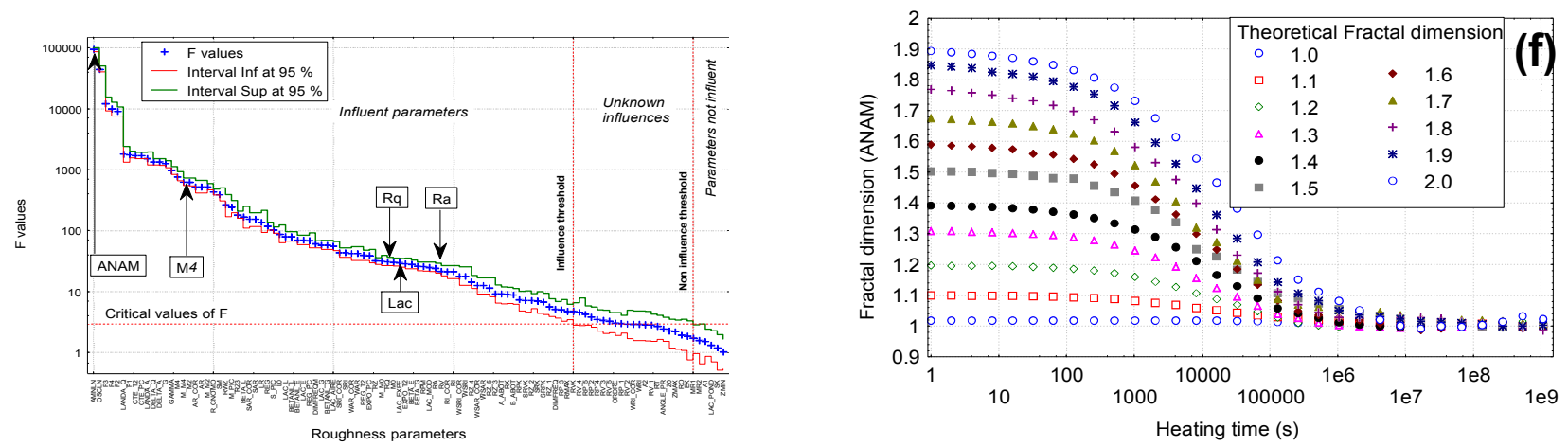

Fig. 3. Left: plot of the bootstrap power discrimination for the roughness parameters of steel sheet. Higher is the F, better is the discrimination of the roughness parameter. Values of each roughness parameter are classified by decreasing order meaning that roughness parameters lies on the left are the best discriminant. The dashed line represents the significant level (at 95\%); under this line, parameter discrimination is statistically irrelevant. Right: Evolution of fractal dimension versus the heating time of an aluminium interface that is submit to a superficial diffusion processes, a volumic one and an evaporation-condensation.

\section{Conclusion}

We have modeled diffusion process on surfaces. The ANAM method allows to well characterize local fractal dimension. With experimental profile, this condition cannot be verified but this hypothesis is not so strong to apply ANAM method on experimental curves. It was shown that fractal dimension was the roughness parameter that better discriminates the diffusion processes.

\section{Acknowledgements}

This work was supported by the Région Picardie (France) and the FEDER (Fonds Européen de Développement Régional) in the Project FoncRug3D.

\section{Appendix 1, Demonstration of the diffusion equation on fractal surface.}

Let now introduce the Weierstrass functions : $\quad z(x, 0)=\sum_{n=0}^{\infty} a_{n} \omega^{-n H} \cos \left(\omega^{n} x+\phi_{n}\right)$

Where $z(x, 0)$ represents the initial profile $z(x, t)$ at diffusion time $t=0, H$ the Hölder exponent ( $H \in[0,2 \pi]), \omega$ a pulse with $\omega>1, a_{n}$ normalized Gaussian random numbers and $\phi_{n}$ uniform random numbers bounded in $[0,2 \pi]$

Although Weierstrass function is nowhere derivative, this function possesses some derivative components. It become then possible to use the theory of partial differential equations of diffusion on each component and then to reconstruct the fractal function. Let $z_{n}(x, 0)$, a component of the Weierstrass function : $\quad z_{n}(x, 0)=a_{n} \omega^{-n H} \cos \left(\omega^{n} x+\phi_{n}\right)$

This component $z_{n}(x, 0)$ will be modified during diffusion time leading to the profile $z_{n}(x, t)$. Such topographical modification of this component $z_{n}(x, t)$ under the superficial diffusion is computed by searching solution of the separate variables $\Phi(x)$ and $\Psi(t)$ such $z_{n}(x, t)=\Phi(x) \Psi(t)$. By derivating Eq.a2 by $t$ and $x$, the following equations are proposed:

$$
\left\{\begin{array}{l}
\partial_{n}(x, t) / \partial=\Phi(x) \Psi^{\prime}(t) \\
\partial z^{4}(x, t) / \partial z^{4}=\Phi^{\prime \prime \prime}(x) \Psi(t)
\end{array}\right.
$$

In the case of superficial diffusion $\frac{\partial z}{\partial t}=-B \frac{\partial^{4} z}{\partial^{4} x}$ (see table 1) and finally, one obtained:

$$
\frac{\Phi^{\prime \prime \prime}(x)}{\Phi(x)}=\frac{\Psi^{\prime}(t)}{\Psi(t)}=-B
$$

As physically $B>0$, then $\Psi^{\prime}(t)=-B \omega^{4 n} \Psi(t)$

$$
\Psi(t)=\exp \left(-B \omega^{4 n} t\right)
$$

and after integration : 
The same mathematical demonstration is applied on volumic and evaporation diffusion (see table 1 for equations) and leads to:

$$
\Psi^{\prime}(t)=-C \omega^{3 n} \Psi(t) \quad(\text { a } 7) \text { and } \Psi^{\prime}(t)=-A \omega^{2 n} \Psi(t)
$$

The profile evolution is due to the combined effects of the three independent diffusion mechanisms: $\Psi^{\prime}(t)=-\left(B \omega^{4 n}+C \omega^{3 n}+A \omega^{2 n}\right) \Psi(t) \quad$ (a9) and then: $\quad \Psi(t)=\exp -\left(B \omega^{4 n}+C \omega^{3 n}+A \omega^{2 n}\right) t \quad$ (a10) The diffusion component at the frequency $\omega_{n}$ is finally: $z_{n}(t, x)=a_{n} \omega^{-n H} \cos \left(\omega^{n} x+\phi_{n}\right) \exp \left(-\left(B \omega^{4 n}+C \omega^{3 n}+A \omega^{2 n}\right) t\right)$

By summing all frequency component, on gets the final equation that models the diffusion on surface:

$$
z(t, x)=\sum_{n=0}^{\infty} a_{n} \omega^{-n H} \cos \left(\omega^{n} x+\phi_{n}\right) \exp \left(-\left(B \omega^{4 n}+C \omega^{3 n}+A \omega^{2 n}\right) t\right)
$$

\section{Appendix 2, The power spectrum of a surface submitted to diffusion processes}

The Fourier transform $\hat{z}_{w, t}$, of the profile defined by equation (Eq.a12) is not defined as a function and could be only defined as a distribution. This leads to $\hat{z}_{w, t}=\sum_{n=0}^{\infty} A_{n, t, \omega}\left(\frac{1}{2} \delta_{\frac{w^{n}}{2 \pi}}+\frac{1}{2} \delta_{\frac{w^{n}}{2 \pi}}\right)$ with $A_{n, t, \omega}=a_{n} \omega^{-n H} \exp \left(-\left(B \omega^{4 n}+C \omega^{3 n}+A \omega^{2 n}\right) t\right)$ where $\delta_{\frac{w^{n}}{2 \pi}}$ is the Dirac impulsion. Let $\gamma_{n}=\frac{w^{n}}{2 \pi}$, a signal frequency, then amplitude spectra is $\mathrm{P}\left[\hat{z}_{w, t}\right]=\sum_{n=0}^{\infty} \frac{A_{n, t \omega}^{2}}{4} \delta_{\gamma_{n}}$

As $\delta_{\gamma_{n}}$ is almost anywhere "null" expect in $\gamma=\gamma_{n}: \mathrm{P}\left[\hat{z}_{\gamma_{p}, t}\right]=\frac{a_{p}^{2}}{4 \gamma_{p}^{2 H}} \exp \left(-2 t\left(B\left(2 \pi \gamma_{p}\right)^{4}+C\left(2 \pi \gamma_{p}\right)^{3}+A\left(2 \pi \gamma_{p}\right)^{2}\right)\right) \sum_{n=0}^{\infty} \delta_{\gamma_{n}}$

This Spectra is always defined as a distribution. It will be of interest to approximate this distribution by a function. Then Dirac impulsion is approximate by a rectangle with width $\left\lfloor\gamma_{p}, \gamma_{p-1}\right\rfloor$ and height $\frac{1}{\gamma_{p}-\gamma_{p-1}}$. As $\gamma_{n}=\frac{w^{n}}{2 \pi}$, discrete variable $n$ becomes a continuous one by letting $n(\gamma) \approx \frac{\log \gamma_{n}+\log 2 \pi}{\log \omega}$. A $\delta n$ variation gives a $\frac{\delta \gamma}{\gamma \log \omega}$ variation. As the smallest element could be only $\delta n=1$, then $\sum_{n=0}^{\infty} \delta_{\gamma_{n}} \approx h(\gamma)=\frac{1}{d \gamma}=\frac{1}{\gamma \log \omega}$. Finally, by mean on the Gaussian for each component, one gets:

$$
\mathrm{P}[\hat{z}(\gamma, t)]=\frac{\mu_{Z}^{2}}{4 \gamma^{2 H+1} \log \omega} \exp \left(-2 t\left(A(2 \pi \gamma)^{2}+C(2 \pi \gamma)^{3}+B(2 \pi \gamma)^{4}\right)\right)
$$

\section{References}

[1] C. Herring: Journal of Applied Physics Vol. 21 (1950), p. 301.

[2] C. Herring, in: Structure and Properties of Solid Surfaces, edited by R. Corner and CS Smith, University of Chicago Press, Chicago, 1953.

[3] W.W. Mullins: Journal of Applied Physics Vol. 28 (1957), p. 333

[4] W.W. Mullins: Journal of Applied Physics Vol. 30 (1959), p. 77.

[5] D. Nicoletti : Chaos, Solitons and Fractals Vol. 5 (1995), 1-8.

[6] J. Lopez, G. Hansali, J.C. Le Bossa and T. Mathia: Journal de physique III Vol. 4 (1994), p. 2501.

[7] C. Tricot: Courbes et dimension fractale, Springer-Verlag, Paris, 1993.

[8] B. Dubuc, J.F. Quiniou, C. Roques-Carnes. C. Tricot and S.W. Zucker: Physical Review A Vol. 39 (1989), p. 1500.

[9] D. Najjar, M. Bigerelle, H. Migaud and A. Iost: Tribology International Vol. 39 (2006), p. 1527.

[10] D. Najjar, M. Bigerelle, F. Hennebelle and A. Iost: Surf. Coat.Tech. Vol. 200 (2006), p. 6088.

[11] S. Ganti and B. Bhushan: Wear Vol. 180 (1995), p. 17.

[12] M. Bigerelle and A. Iost: Computer and mathematics with applications Vol. 42 (2001), p. 241. 